\title{
Influence of Deformation on CVC p-n-Junction in a Strong Microwave Field
}

\author{
Muhammadjon Gulomkodirovich Dadamirzayev \\ Namangan Engineering Pedagogical Institute, Namangan, Uzbekistan \\ Email: gulyamov1949@mail.ru
}

Received 2 February 2015; accepted 16 February 2015; published 25 February 2015

Copyright @ 2015 by author and Scientific Research Publishing Inc.

This work is licensed under the Creative Commons Attribution International License (CC BY). http://creativecommons.org/licenses/by/4.0/

c) (i) Open Access

\begin{abstract}
This paper investigates the current-voltage characteristics (CVC) strain of p-n-junction in a strong microwave (MW) field and shows that the deformation increases the current generated in the p-njunction. We analyze the current-voltage characteristics of $p$-n-junction in which three-dimensional space $(I, U, \varepsilon)$ gives more complete information than the two-dimensional.
\end{abstract}

\section{Keywords}

Microwave Electromagnetic Field, Deformation Effects in Semiconductors, The Concentration of Minority Carriers, The Current-Voltage Characteristic of the p-n-Junction, The Temperature of the Electrons and Holes

\section{Introduction}

The first experimental study of the heating of the charge carriers by the strong electric field in inhomogeneous semiconductors began with thermopower measurements of hot charge carriers occurring at the p-n-junction. [1] [2] studied the current-voltage characteristics (CVC) and investigated the variation of photoelectric characteristics of silicon p-n-junctions in strong microwave fields. And in [3] [4], the results of theoretical and experimental studies of the effect of occurrence of negative differential resistance of the diode structures based on p-njunction theoretically described experimentally observed effect of occurrence of negative differential resistance mode and switch to the tunnel diode when exposed to high levels of microwave power. EMF of hot carriers $U_{o c}$, generated at unsymmetrical p-n-junction in the microwave electromagnetic field, despite the fact that the electron temperature much higher than that of the holes is determined by the hot holes [5]. In [6] theoretically studied the effect of lattice heating on the kinetics of the establishment of the thermopower of hot carriers in the p-n-junction. It is shown that the heating of the lattice leads to an additional third stage of establishing thermoelectric current and thermopower of hot carriers with a relaxation time determined by the thermal conductivity 
and heat capacity of the sample. In [7] studied the effect of distortion of the heating wave on the recombination currents and electromotive force generated at the p-n-junction in a strong microwave field. It is shown that high-frequency perturbation of the surface potential and the height of the p-n-junction in the mode of the short circuit current decreases the effective barrier height, and idling to anomalously large values of the EMF. In addition, the effect of deformation on the electrical properties of semiconductor devices was presented in many papers [8]-[10], where it showed the action of the pressure change-voltage characteristic of p-n-junctions, Schottky diodes, transistors and other semiconductor devices. The study of these phenomena is of interest from two points of view. Firstly, changes in the characteristics of semiconductor devices can be used for indicating pressure or other mechanical quantities in pressure sensors, audio receivers and similar devices. Secondly, these phenomena are of interest in terms of the definition of the physical processes causing the reliability of semiconductor devices, as the deformation occurring in the unit, for whatever reasons, lead to disturbances in his work. However, the above studies did not investigate the influence of deformation on the CVC p-n-junction in a strong microwave field.

According to the concept of elementary excitations of a semiconductor is the electron-hole and phonon gas. Electromagnetic wave (microwave, light) excites an electron-hole and phonon system. In the pulsed mode of microwave waves or light mainly heats the electron-hole system. Phonon system remains unexcited. When the impact of variable deformation excited phonon system, and the electron-hole system is rebuilt to the changes of the crystal lattice. Thus, the microwave and the deformation from different sides excites semiconductor systems, from the electron-hole and phonon system. Investigation using the microwave [1]-[7] and the variable deformation [8]-[10] allows two different sides examine the semiconductor structure.

Investigation of the effect of deformation on the current and voltage in strong microwave fields of interest, both from a physical point of view, and in the practical application. When the strain changes the energy spectrum, in particular, the band gap can change the saturation currents of p-n-junction. This can greatly affect the currents generated in the p-n-junction of the microwave field. On the other hand, p-n-transitions in strong microwave fields may often be exposed to strong mechanical vibration. It may also affect the current and EMF generated by p-n-junction. You can expect a change CVC p-n-junction with simultaneous exposure to the microwave field and deformation.

However, the previous studies [1]-[10] did not consider the simultaneous influence of mechanical deformation and the microwave field at the CVC p-n-junction.

The aim of this work is to study the effect of deformation on the CVC p-n-junction in a strong microwave field.

\section{Calculations of the CVC Strain of p-n-Junction in a Strong Microwave Field}

The average value of the total current through the diode under the influence of an electromagnetic wave consists of the electron and hole currents [5].

At high power wave when $T_{e} \neq T_{h} \neq T, \mathrm{CVC}$ is determined by the formula [5]:

$$
\begin{aligned}
\bar{j}= & j_{\text {se }}\left\{\left(\frac{T_{e}}{T}\right)^{\frac{1}{2}} \exp \left(\frac{e \varphi_{0}}{k T}-\frac{e\left(\varphi_{0}-U\right)}{k T_{e}}\right) \int_{0}^{2 \pi} \exp \left(-\frac{e U_{B} \cos (\omega t)}{k T_{e}}\right) \frac{\mathrm{d}(\omega t)}{2 \pi}-1\right\} \\
& +j_{s h}\left\{\left(\frac{T_{h}}{T}\right)^{\frac{1}{2}} \exp \left(\frac{e \varphi_{0}}{k T}-\frac{e\left(\varphi_{0}-U\right)}{k T_{h}}\right) \int_{0}^{2 \pi} \exp \left(-\frac{e U_{B} \cos (\omega t)}{k T_{h}}\right) \frac{\mathrm{d}(\omega t)}{2 \pi}-1\right\}
\end{aligned}
$$

where, $j_{s e}=\frac{e D_{e} n_{p}}{L_{e}}, j_{s h}=\frac{e D_{h} p_{n}}{L_{h}}$-saturation currents of electrons and holes; $\varphi_{0}$-height of the potential barrier in the absence of an electromagnetic wave; $\varphi=\varphi_{0}-U ; U$-the resulting voltage across the diode; $U_{B}=-\int_{0}^{d} E_{\mathrm{B}} \mathrm{d} x-\mathrm{AC}$ voltage of the incident wave, created by the barrier diode; $T$ is the temperature of the lattice; $k$-Boltzmann constant; $T_{e}$ and $T_{h}$-temperature of electrons and holes; $E_{B}$ —electric field of the wave; e is the charge of the electron; $D_{e}$ and $D_{h}$-diffusion coefficients of electrons and holes, $L_{e}$ and $L_{h}-$ 
their diffusion length; $n_{p}$ and $p_{n}$-the concentration of minority carriers.

If the value of the diffusion coefficient, diffusion length, mobility and concentration of electrons and holes for a silicon p-n-junction to take as in [5] [11], and given the dependence of the intrinsic concentration of charge carriers from the lattice temperature and the band gap of silicon [11]:

$$
\begin{aligned}
n_{i}^{2} & =1.5 \times 10^{33} \times T^{3} \times \mathrm{e}^{-\frac{\Delta \varepsilon}{k T}} \\
\Delta \varepsilon & =1.27 \ni \mathrm{B}
\end{aligned}
$$

then the formula (1) can be written as follows:

$$
\begin{aligned}
\bar{j}= & \sqrt{\frac{e k T_{e}}{\tau_{e}} \times T^{3.4} \times 10^{9}} \times \frac{3 \times 10^{33} \times \mathrm{e}^{-\frac{\varepsilon_{g}}{k T}}}{p_{p}} \\
& \times\left\{\left(\frac{T_{e}}{T}\right)^{\frac{1}{2}} \exp \left(\frac{e \varphi_{0}}{k T}-\frac{e\left(\varphi_{0}-U\right)}{k T_{e}}\right) \int_{0}^{2 \pi} \exp \left(-\frac{e U_{B} \cos (\omega t)}{k T_{e}}\right) \frac{\mathrm{d}(\omega t)}{2 \pi}-1\right\} \\
& +\sqrt{\frac{e k T_{h}}{\tau_{h}} \times T^{3.7} \times 2.5 \times 10^{8}} \times \frac{1.5 \times 10^{33} \times e^{-\frac{\varepsilon_{g}}{k T}}}{n_{n}} \\
& \times\left\{\left(\frac{T_{h}}{T}\right)^{\frac{1}{2}} \exp \left(\frac{e \varphi_{0}}{k T}-\frac{e\left(\varphi_{0}-U\right)}{k T_{h}}\right) \int_{0}^{2 \pi} \exp \left(-\frac{e U_{B} \cos (\omega t)}{k T_{h}}\right) \frac{\mathrm{d}(\omega t)}{2 \pi}-1\right\}
\end{aligned}
$$

If in this equation will take into account permanent deformation that:

$$
\varepsilon_{g}(\varepsilon, T)=\varepsilon_{g}(0)+\Delta \varepsilon
$$

Then the formula (4) has the following form:

$$
\begin{aligned}
\bar{j}= & \sqrt{\frac{e k T_{e}}{\tau_{e}} \times T^{3.4} \times 10^{9}} \times \frac{3 \times 10^{33} \times \mathrm{e}^{-\frac{\varepsilon_{g}(0)+\Delta \varepsilon}{k T}}}{p_{p}} \\
& \times\left\{\left(\frac{T_{e}}{T}\right)^{\frac{1}{2}} \exp \left(\frac{e \varphi_{0}}{k T}-\frac{e\left(\varphi_{0}-U\right)}{k T_{e}}\right) \int_{0}^{2 \pi} \exp \left(-\frac{e U_{B} \cos (\omega t)}{k T_{e}}\right) \frac{\mathrm{d}(\omega t)}{2 \pi}-1\right\} \\
& +\sqrt{\frac{e k T_{h}}{\tau_{h}} \times T^{3.7} \times 2.5 \times 10^{8}} \times \frac{1.5 \times 10^{33} \times \mathrm{e}^{-\frac{\varepsilon_{g}(0)+\Delta \varepsilon}{k T}}}{n_{n}} \\
& \times\left\{\left(\frac{T_{h}}{T}\right)^{\frac{1}{2}} \exp \left(\frac{e \varphi_{0}}{k T}-\frac{e\left(\varphi_{0}-U\right)}{k T_{h}}\right) \int_{0}^{2 \pi} \exp \left(-\frac{e U_{B} \cos (\omega t)}{k T_{h}}\right) \frac{\mathrm{d}(\omega t)}{2 \pi}-1\right\}
\end{aligned}
$$

Using formula (6), first consider the CVC p-n-junction for different strains (Figure 1). From these results it follows that with increasing strain the current $\mathrm{p}$-n-junction increases.

Now consider using the formula (6) CVC p-n-junction (Figure 2): 1) without distortion and the microwave field (when $\varepsilon=0 ; T=300 \mathrm{~K} ; T_{e}=300 \mathrm{~K} ; T_{h}=300 \mathrm{~K} ; U_{B}=0.2 \mathrm{~B} ; \varphi_{0}=0.5 \mathrm{~B} ; \tau_{e}=10^{-9} \mathrm{c} ; \tau_{h}=10^{-7} \mathrm{c}$; $\varepsilon_{q}=1.21 \ni \mathrm{B}$ ); 2) under strain without the microwave field (when $\varepsilon=10^{-3} ; T=300 \mathrm{~K} ; T_{e}=300 \mathrm{~K} ; T_{h}=300$ $\left.\mathrm{K} ; U_{\mathrm{B}}=0.2 \mathrm{~B} ; \varphi_{0}=0.5 \mathrm{~B} ; \tau_{e}=10^{-9} \mathrm{c} ; \tau_{h}=10^{-7} \mathrm{c} ; \varepsilon_{g}=1.21 \mathrm{\jmath B}\right)$; 3) without distortion at microwave exposure (when $\varepsilon=0 ; T=300 \mathrm{~K} ; T_{e}=500 \mathrm{~K} ; T_{h}=400 \mathrm{~K} ; U_{B}=0.2 \mathrm{~B} ; \varphi_{0}=0.5 \mathrm{~B} ; \tau_{e}=10^{-9} \mathrm{c} ; \tau_{h}=10^{-7} \mathrm{c}$; $\varepsilon_{g}=1.21 \ni \mathrm{B}$ ); 4) upon deformation and microwave exposure (where $\varepsilon=10^{-3} ; T=300 \mathrm{~K} ; T_{e}=500 \mathrm{~K}$; $\left.T_{h}=400 \mathrm{~K} ; U_{B}=0.2 \mathrm{~B} ; \varphi_{0}=0.5 \mathrm{~B} ; \tau_{e}=10^{-9} \mathrm{c} ; \tau_{h}=10^{-7} \mathrm{c} ; \varepsilon_{g}=1.21 \mathrm{\jmath B}\right)$.

Figure 2 shows that the strain increases currents through the p-n-junction in the absence of the microwave field (Figure 2, curves 1, 2) and under strong heating (Figure 2, curves 3, 4) of electrons and holes. 


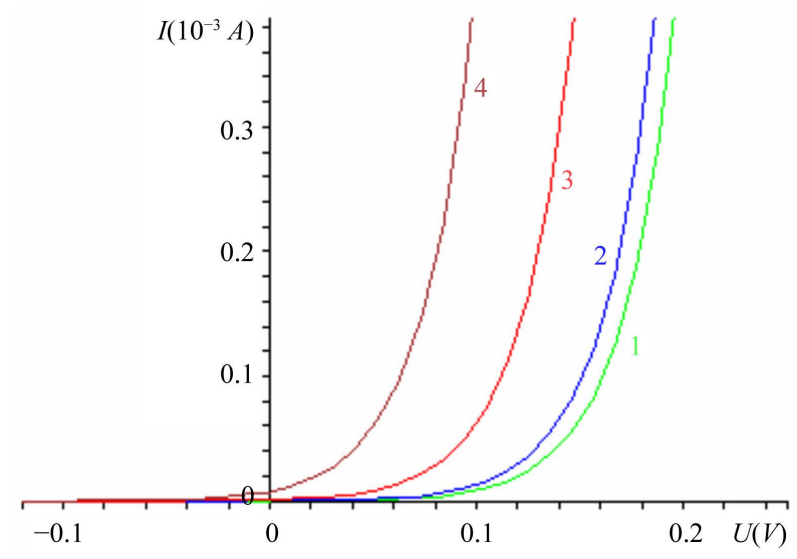

Figure 1. CVC p-n-junction under strain: $T_{e}=300 \mathrm{~K}, T_{h}=300$ $\mathrm{K} ; 1-\varepsilon=0 ; 2-\varepsilon=0.001 ; 3-\varepsilon=0.005 ; 4-\varepsilon=0.01$.

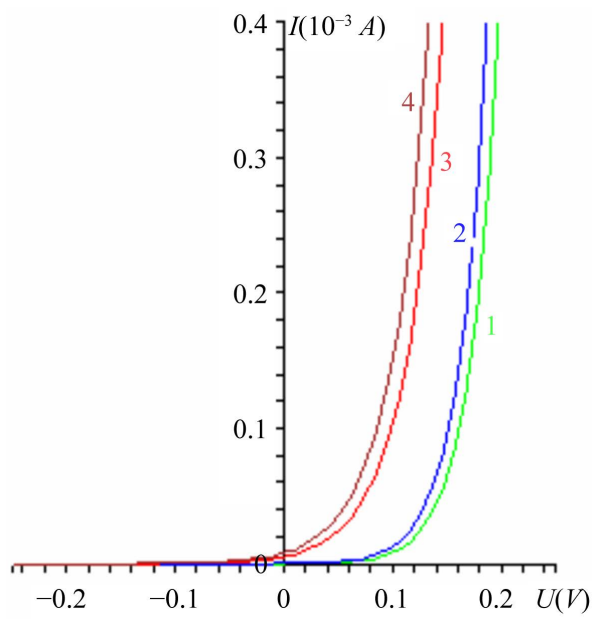

Figure 2. CVC p-n-junction: 1-without distortion and the microwave field; 2 - under strain without the microwave field; 3 -without distortion at microwave exposure; 4-upon deformation and microwave exposure.

Figure 3 shows the IV characteristics of p-n-junction in three-dimensional space $I, U, \varepsilon$ : without microwave (when $T_{e}=300 \mathrm{~K} ; T_{h}=300 \mathrm{~K}$ field); when exposed to microwave (when $T_{e}=500 \mathrm{~K} ; T_{h}=400 \mathrm{~K} ; T_{e}=$ $600 \mathrm{~K} ; T_{h}=500 \mathrm{~K}$ ) field.

We showed that as the microwave is free and deformation increases currents of hot carriers in the p-n-junction. This is due to the fact that the microwave effect on the electron-hole gas, and the deformation changes the energy structure of semiconductors. It turned out that even in a strong microwave field deformation increases currents at the p-n-junction. This is due to the fact that the currents of p-n-junction affect both the heating of the electron-hole gas and the change in the deformation of the band gap of the semiconductor.

\section{Conclusions}

Firstly, we examined voltage-current characteristic strain p-n junction in a strong microwave field. The variation of the band gap semiconductor due to deformation influences the concentration of minority carriers in p-n junction. This leads to increase saturation currents of the diode. The strongly changing of the saturation increases the currents of hot electrons and holes through the p-n junction. It is shown that the deformation increases the currents generated in the p-n junction which is located in the microwave field.

We analyzed current-voltage characteristics of p-n transition in three-dimensional space $(I, U, \varepsilon)$. This gives 


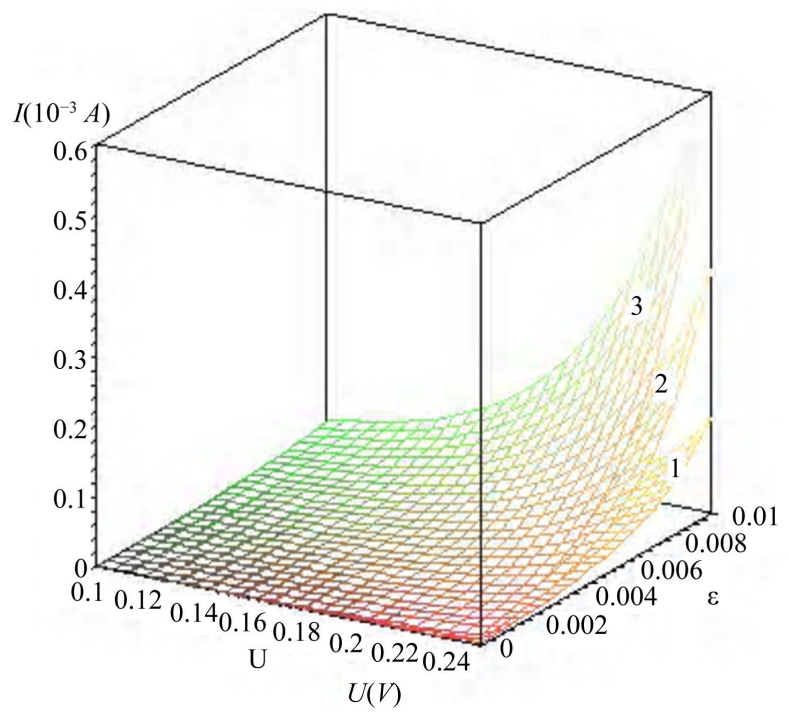

Figure 3. CVC p-n-junction in the three $I, U, \varepsilon$ space: $1-$ no microwave (when $T_{e}=300 \mathrm{~K} ; T_{h}=300 \mathrm{~K}$ ) field; 2 -when exposed to microwave (when $T_{e}=500 \mathrm{~K} ; T_{h}=400 \mathrm{~K}$ ) field; 3 - when exposed to microwave (when $T_{e}=600 \mathrm{~K} ; T_{h}=500 \mathrm{~K}$ ) field.

more complete information about the mode of deformation of the diode under various values in a strong microwave field.

\section{References}

[1] Ablyazimova, N.A. and Veynger, A.I. (1988) Semiconductors, 22, S2001-S2007.

[2] Ablyazimova, N.A. and Veynger, A.I. (1992) Semiconductors-St. Petersburg, 26, S1041-S1047.

[3] Usanov, D.A., Skripal, A.V. and Ugryumova, N.V. (1998) Semiconductors, 32, S1399-S1402.

[4] Usanov, D.A., Skripal, A.V., Ugryumova, N.V., Venig, S.B. and Orlov, V.E. (2000) Semiconductors, 34, S567-S571.

[5] Dadamirzaev, M.G. (2011) Semiconductors-St. Petersburg, 45, 299-302.

[6] Gulyamov, G., Dadamirzaev, M.G. and Boydedaev, S.R. (2000) Semiconductors—St. Petersburg, 34, 266.

[7] Gulyamov, G., Dadamirzaev, M.G. and Boydedaev, S.R. (2000) Semiconductors—St. Petersburg, 34, 572.

[8] Polyakova, A.L. (1979) Deformation of Semiconductors and Semiconductor Devices.

[9] Polyakov, A. (1972) Acoustical Physics, 18, 1-22.

[10] Bahadyrhanov, M.K. and Abdurahimov, A. (1987) FTP, 21, S1710-S1712.

[11] Baransky, P.I., Klochkov, V.P. and Potykevich, I.V. (1975) Semiconductor Electronics. Kyiv., Naukova Dumka, 704. 
Scientific Research Publishing (SCIRP) is one of the largest Open Access journal publishers. It is currently publishing more than 200 open access, online, peer-reviewed journals covering a wide range of academic disciplines. SCIRP serves the worldwide academic communities and contributes to the progress and application of science with its publication.

Other selected journals from SCIRP are listed as below. Submit your manuscript to us via either submit@scirp.org or Online Submission Portal.
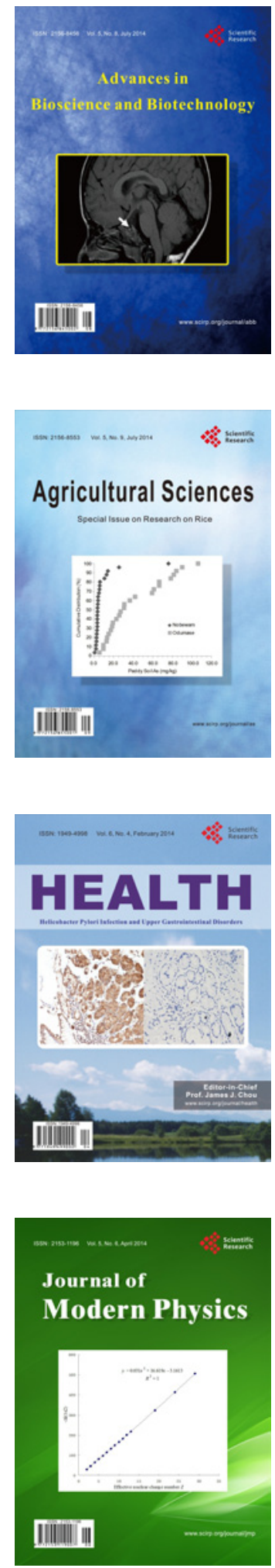
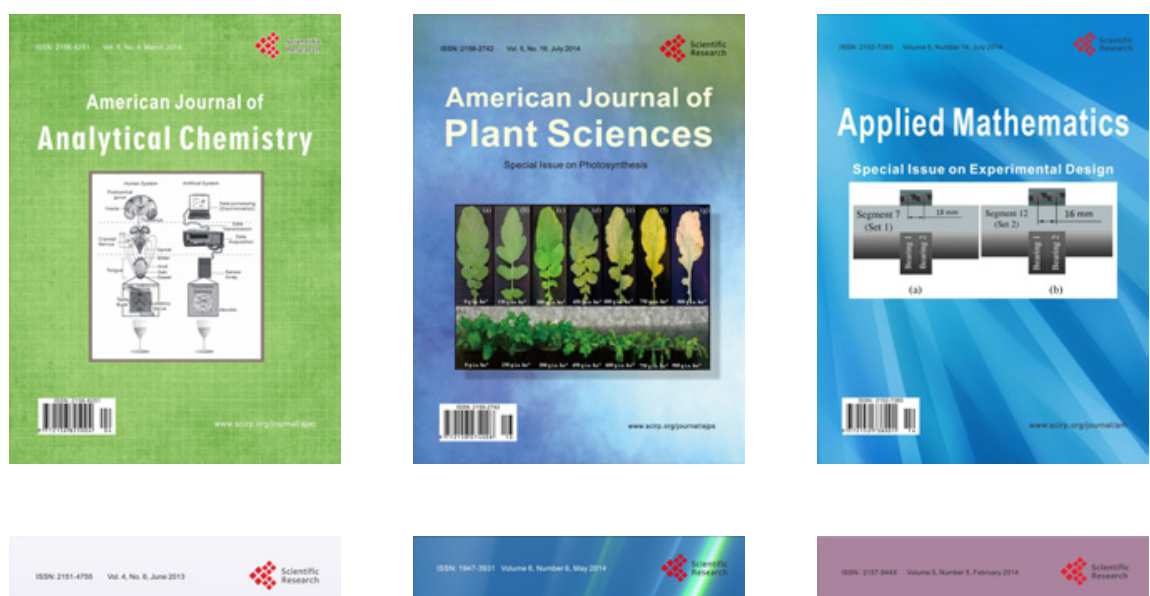

Creative Education
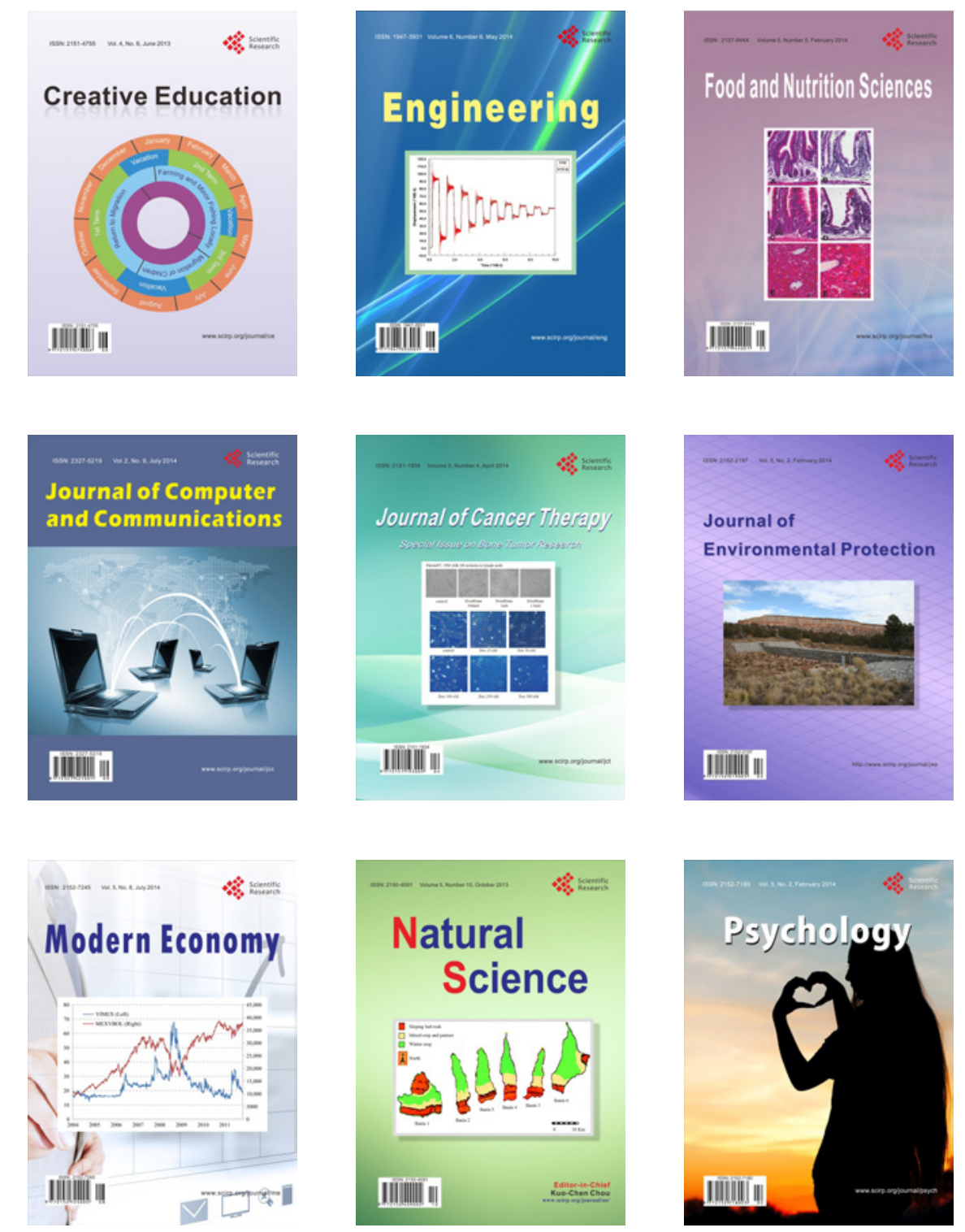